\title{
RECUPERAÇÃO AVANÇADA DE PETRÓLEO: AVALIAÇÃO DE UM AGENTE QUELANTE EM SOLUÇÕES MICELARES DE TENSOATIVO ANIÔNICO NA PRESENÇA DE SAIS
}

\author{
J. C. C. MOURA JÚNIOR ${ }^{1}$, F. K. G. SANTOS ${ }^{1}$, J. S. CARVALHO SANTOS ${ }^{2}$, S. K. S. NUNES ${ }^{2}$, E. \\ L. BARROS NETO' ${ }^{2}$ R. H. L. LEITE ${ }^{1}$ \\ ${ }^{1}$ Universidade Federal Rural do Semi-Árido, Departamento de Agrotecnologia e Ciências Sociais \\ ${ }^{2}$ Universidade Federal do Rio Grande do Norte, Departamento de Engenharia Química \\ E-mail para contato: jose.carlos_network@hotmail.com
}

\begin{abstract}
RESUMO - Uma das maiores barreiras que dificultam a recuperação avançada de petróleo é a alta tensão interfacial entre o fluido injetado e o óleo do reservatório. O uso de tensoativo diminui a tensão interfacial óleo/fluido injetado, no entanto os tensoativos aniônicos interagem com os sais presentes na água. Nesse sentido, este trabalho tem como objetivo avaliar os efeitos das soluções de tensoativos com e sem a presença de um agente quelante na recuperação avançada. Para simular um reservatório de petróleo foi utilizado um arenito da formação Botucatu. Os testemunhos foram preparados em formato cilíndrico. Foram injetados água salgada e petróleo. A recuperação convencional foi realizada com água salgada. A recuperação avançada foi com solução micelar de um tensoativo aniônico. Foram avaliadas recuperações com e sem o agente quelante. De acordo com os resultados, observou-se uma pequena diferença na fração de recuperação onde se usou o agente quelante.
\end{abstract}

\section{INTRODUÇÃO}

A recuperação avançada de petróleo é tida como um importante caminho na indústria do óleo e gás, principalmente no momento atual onde as fontes de energia fóssil estão cada vez mais caras tanto na descoberta quanto na produção (Khosravani et al., 2013). Alguns campos petrolíferos do Kuwait, por exemplo, sofreram esgotamento natural durante a etapa de injeção de água. Depois da fase de extração secundária, a quantidade de água é rapidamente aumentada e a recuperação diminuída. Mais da metade do óleo ainda está no campo petrolífero. Portanto, métodos de recuperação terciária deveriam ser aplicados (Yin e Zhang, 2013).

Outro fator que diminui a recuperação de petróleo é a alta tensão interfacial entre os fluidos. Para solucionar esse problema, é injetado o tensoativo que tem propriedade anfifílica e atua na interface entre a água e o óleo aumentando o deslocamento do petróleo e, dessa forma, o fator de recuperação (Thomas, 2001).

As propriedades dos tensoativos diferem de moléculas não-anfifílicas em um principal aspecto: devido às mudanças abruptas nas suas propriedades quando estão acima da concentração crítica. 


\section{9 a 22 de outubro de 2014 \\ Florianópolis/SC}

Abaixo dessa concentração, a maioria das propriedades é similar às de um simples eletrólito. Uma característica notável é que a tensão superficial diminui rapidamente com o aumento da concentração do tensoativo. Contudo, todas as propriedades mostram uma abrupta mudança a uma concentração particular que consiste no fato de que, acima dessa concentração, as superfícies ativas dos íons ou moléculas em solução se associam para formar unidades maiores. Estas unidades associadas são chamadas de micelas. A concentração na qual esse fenômeno de associação ocorre é conhecida como concentração micelar crítica (c.m.c) (Ahmadi et al., 2013).

A ação dos tensoativos pode ser influenciada por alguns fatores como salinidade. Cada tensoativo tem uma c.m.c. a uma dada temperatura e concentração eletrolítica (Ahmadi e Shadizaeh, 2013). A adição de sal produz uma redução da c.m.c., sendo possível que essa redução seja de até 100 vezes menor para alguns tensoativos. A partir da c.m.c., há formação de agregados conhecidos como micelas e a adição de tensoativo não reduz mais a tensão interfacial (Daltin, 2011). O EDTA ou ácido etilenodiamino tetra-acético é um composto orgânico que age como agente quelante, formando complexos muito estáveis com diversos íons metálicos presentes na solução micelar.

Chen et al. (2013) realizaram testes para recuperar petróleo pesado pelo uso de álcali combinado com tensoativo. Observou-se um efeito sinergético entre o tensoativo e o álcali. O tensoativo usado foi o poliglicósido de alquila (chamado de APG) e obteve uma tensão interfacial entre o petróleo e a água de $10^{-2} \mathrm{mN} / \mathrm{m}$. Quando foi adicionado carbonato de sódio $(0,5 \%)$ e cotensoativo sulfonato de petróleo de Shengli, denominado de SLPS - Shengli petroleum sulfonate $(0,03 \%)$ juntamente com o tensoativo, a tensão interfacial diminuiu ainda mais, chegando a $10^{-4} \mathrm{mN} / \mathrm{m}$ com recuperação avançada de 19,4\%. Esses valores foram encontrados quando a concentração do APG foi de $0,05 \%$. Percebeu-se que tensão interfacial excessivamente baixa diminui o fator de recuperação, pois $0,05 \%$ de $\mathrm{APG}, 0,5 \%$ de $\mathrm{Na}_{2} \mathrm{CO}_{3}$ e $0,1 \%$ de SLPS com tensão interfacial de $7,23 \times 10^{-5} \mathrm{mN} / \mathrm{m}$ obtiveram $15,1 \%$ na recuperação avançada.

Pei et al. (2014) investigaram o efeito do polímero poliacrilamida parcialmente hidrolisada e do $\mathrm{NaOH}$ na interação com óleo pesado do campo petrolífero de Shengli, da China, na recuperação avançada de petróleo. Foi testado primeiramente o polímero e percebeu-se que a ação dele sozinho é limitada. Posteriormente, foi utilizada a poliacrilamida e o hidróxido de sódio e houve um efeito sinergético no qual a recuperação do petróleo pesado foi melhorada. Porém, eles concluíram que a ação dos dois na recuperação foi mais eficiente do que se algum deles fosse usado isoladamente em cada recuperação. Obtiveram-se melhores resultados quando se injetou polímero e base alternadamente, e não ao mesmo tempo.

Baseado no exposto, esse trabalho tem o objetivo de avaliar o comportamento da solução micelar do tensoativo aniônico SB (sabão base) e, também, da injeção dessa solução micelar com um agente quelante, EDTA, assim como sua interação com solução salina, na recuperação avançada de petróleo. 


\section{METODOLOGIA}

\subsection{Materiais e Reagentes}

Foram utilizados os seguintes equipamentos: Balança (Modelo KB 8000, marca Ind. e Com. Eletrônica Gehaka Ltda); Mufla; Espectofotômetro (Modelo Cary50, marca Varian); Sistema de injeção (holder); Centrífuga (Marca Quimis); Tensoativo aniônico SB 30\% acima da c.m.c; Cloreto de cálcio a $0,2 \%$; Cloreto de potássio a 2\%; Óleo mineral.

\subsection{Metodologia}

Pesaram-se os plugs secos e molhados para determinar a porosidade da rocha. Foi escolhido o plug mais adequado para a recuperação. Em seguida, o plug foi introduzido no holder onde se montou o sistema de injeção, conforme mostrado na Figura 1.

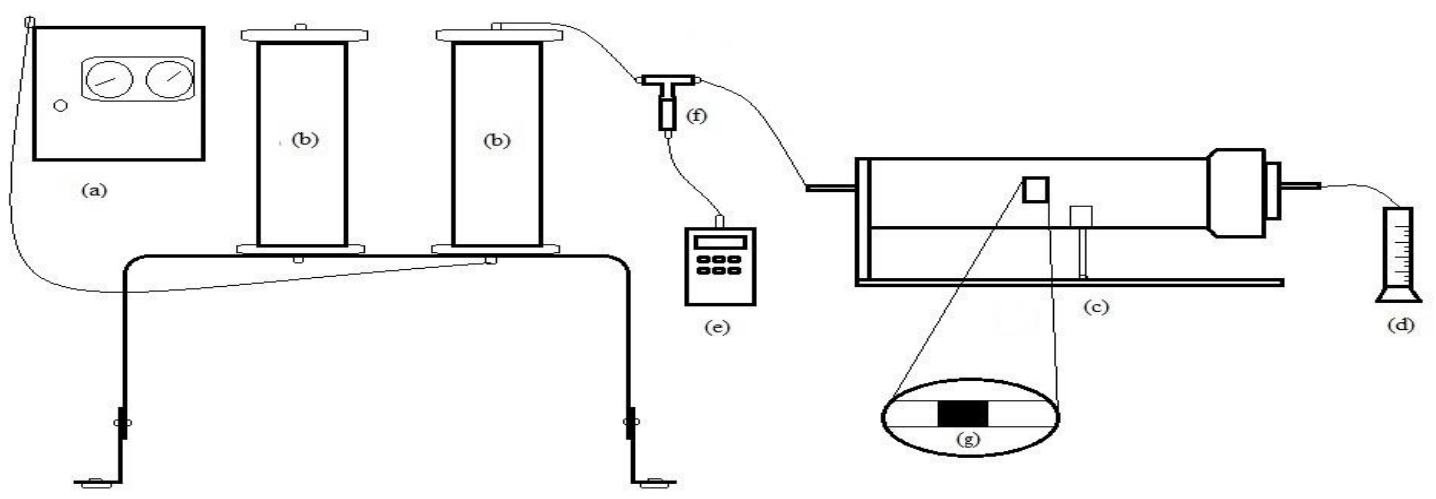

Figura 1 - Sistema de injeção: (a) bomba, (b) células que contêm o petróleo, a solução salina e o tensoativo, (c) holder que contém o plug, (d) proveta, (e) barômetro, (f) transdutor de pressão, (g) plug dentro do holder.

A solução salina foi preparada em um balão volumétrico de $2 \mathrm{~L}$ com água destilada, cloreto de potássio $(\mathrm{KCl})$ a $2 \%$ e cloreto de cálcio $\left(\mathrm{CaCl}_{2}\right)$ a $0,2 \%$.

A recuperação foi dividida em quatro etapas: saturação da rocha com solução salina (primeira etapa), saturação da rocha com petróleo cru (segunda etapa), recuperação convencional (terceira etapa), recuperação avançada (quarta etapa). Na primeira etapa, injetou-se a solução salina anotando a pressão a cada minuto até o décimo minuto. Depois disso, a pressão foi verificada a cada 10 minutos.

Em seguida, na segunda etapa iniciou-se a injeção do petróleo onde ele foi coletado em 5 provetas de $10 \mathrm{~mL}$. Nos primeiros 15 minutos, a pressão foi medida a cada minuto. Posteriormente, a pressão foi medida a cada 10 minutos até completar as 5 provetas.

Na terceira etapa foram separados 20 tubos de ensaios para a recuperação simples. A cada 5 minutos a pressão foi verificada e um novo tubo de ensaio foi recolocado para coletar o petróleo 
deslocado pela salmoura até o vigésimo tubo.

$\mathrm{Na}$ quarta etapa tem-se a recuperação avançada injetando o tensoativo SB $30 \%$ acima da c.m.c. Utilizaram-se os mesmos procedimentos da terceira etapa.

Posteriormente, foi adicionado querosene em todos os tubos de ensaio das recuperações simples e avançada. Eles foram tampados com papel filme e agitados com o querosene para depois serem centrifugados.

Com a micropipeta foram retirados volumes pequenos, em geral, dependendo da concentração do óleo. Esse volume foi colocado num balão volumétrico de $10 \mathrm{~mL}$ sendo completado com querosene e agitado. Em seguida todas as amostras foram colocadas na cubeta e analisadas para obter a absorbância.

Foram feitas três recuperações. Na primeira, usando solução micelar de tensoativo SB 30\% acima da c.m.c. Na segunda, usou-se a solução micelar até o $12^{\circ}$ volume poroso, no restante, foi usada a solução salina novamente. Na terceira, tensoativo SB com EDTA a 0,01M.

Na primeira recuperação o plug utilizado tinha diâmetro de $40 \mathrm{~mm}$ e altura de $30 \mathrm{~mm}$. Já na segunda recuperação as dimensões foram $41 \mathrm{~mm}$ de diâmetro e $31,2 \mathrm{~mm}$ de altura.

Foi usado o porosímetro a nitrogênio para determinar a porosidade da rocha. Uma curva de calibração foi feita a partir do volume poroso conhecido em função da pressão. Sendo o coeficiente de correlação aproximadamente 1 , a porosidade da rocha $(\phi)$ foi calculada pela relação entre o volume poroso ou espaços vazios e o volume total, conforme a Equação 1:

$$
\phi=\frac{\text { Volume Poroso }}{\text { Volume Total }}
$$

O fator de recuperação total foi determinado pela soma do fator de recuperação convencional, onde a massa de petróleo recuperada é obtida pelo balanço de massa, com o fator de recuperação da avançada, onde a massa de petróleo foi obtida pelo espectofotômetro, como mostrado na Equação 2:

$$
F R_{t}=F R_{g}+F R_{a}=\frac{M_{a}+M_{\varpi}}{M_{\text {tinj } j}}
$$

onde $\mathrm{FR}_{\mathrm{t}}$ é o fator de recuperação total, $\mathrm{FR}_{\mathrm{s}}$ é o fator de recuperação na recuperação simples, $\mathrm{FR}_{\mathrm{a}}$ é o fator de recuperação na recuperação avançada, $M_{\mathrm{s}}$ é a massa de petróleo recuperada na recuperação simples, $\mathbf{M}_{\mathrm{a}}$ é a massa de petróleo recuperada na recuperação avançada e $\mathbf{M}_{\mathrm{tinj}}$ é a massa total injetada.

\section{RESULTADOS E DISCUSSÃO}

Extraiu-se aproximadamente $50 \mathrm{~mL}$ de petróleo na saturação do petróleo. Cerca de $2 \mathrm{~mL}$ de 
solução salina foram extraídas também porque ela já estava presente na rocha devido à saturação com água salina. Esse processo de saturação com óleo e água salgada foi utilizado nas três recuperações e serve para tornar o sistema semelhante a um poço, onde se encontra o petróleo e a solução salina.

Foi retirada uma alíquota da fase superior de cada tubo de ensaio e, em seguida, a quantidade retirada de petróleo foi diluída para baixar a concentração para ser analisada no espectofotômetro.

A porosidade da rocha da primeira, segunda e terceira recuperação foi de $0,1936,0,2283$ e 0,1969 respectivamente, de acordo com a Equação 1.

A Figura 2 mostra o resultado do fator de recuperação com o volume poroso para a primeira recuperação avançada.

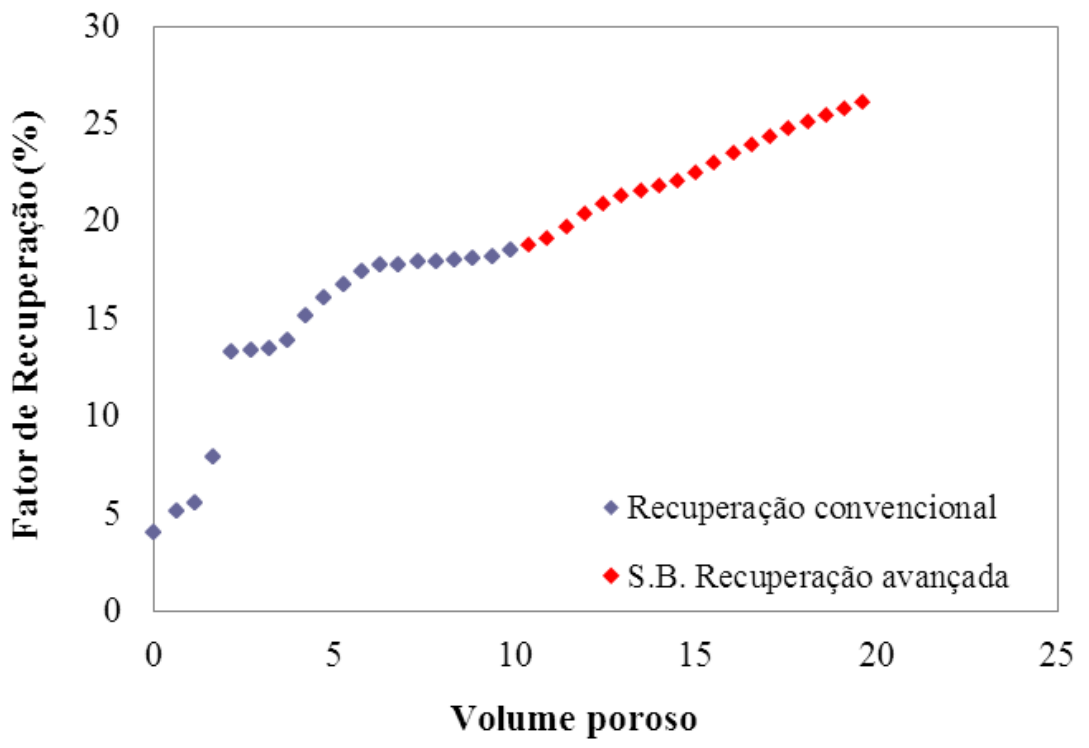

Figura 2 - Relação entre o fator de recuperação e o volume poroso, usando apenas o tensoativo SB na recuperação avançada.

Analisando a Figura 2, pode-se perceber que nessa recuperação injetou-se aproximadamente 10 VP (volumes porosos) de solução salina de cloreto de potássio e cloreto de cálcio para obter um percentual de óleo na recuperação convencional de 18,54\%; sendo a densidade do petróleo usado de $0,8675 \mathrm{~g} / \mathrm{cm}^{3}$, com a pressão inicial e final de 0,555 bar e 0,539 bar, respectivamente. Já na recuperação avançada injetou-se aproximadamente 9 VP de solução de tensoativo aniônico SB para obter uma porcentagem de $7,54 \%$, sendo a pressão inicial e final de $0,450 \mathrm{bar}$ e $0,825 \mathrm{bar}$, respectivamente. A recuperação total foi de $26,09 \%$.

A Figura 3 mostra o resultado do fator de recuperação com o volume poroso para a segunda recuperação avançada. 


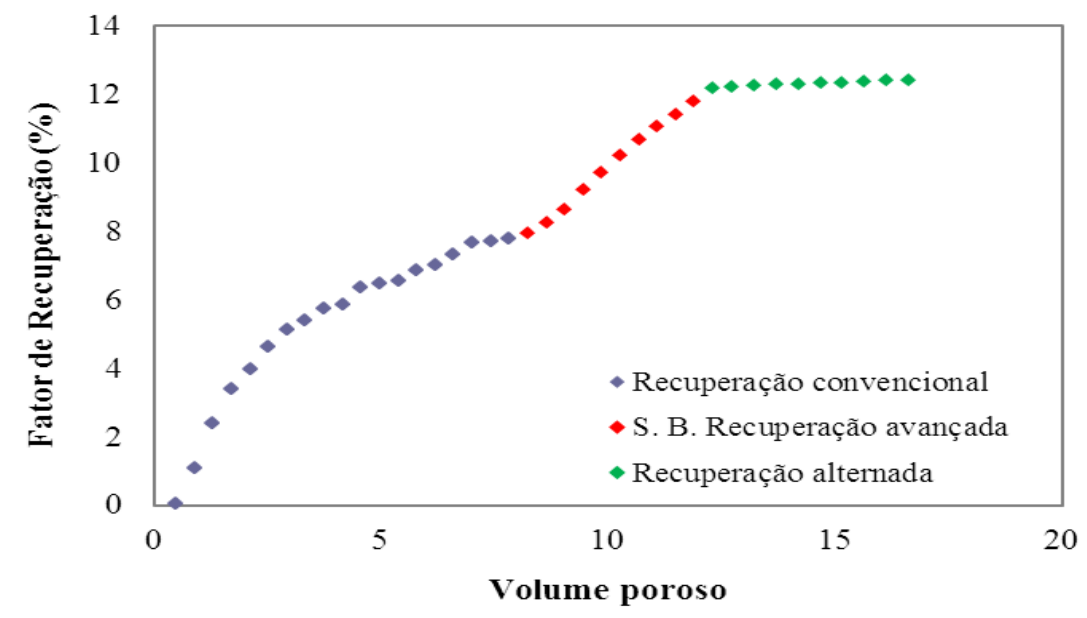

Figura 3 - Relação entre o fator de recuperação e o volume poroso, usando o tensoativo SB até o décimo segundo VP, sendo os demais com salmoura.

Analisando a Figura 3, pode-se perceber que o percentual de óleo na recuperação simples foi de $7,95 \%$, com a utilização de $8 \mathrm{VP}$ de solução salina. A pressão inicial e final nessa etapa foi de 0,235 bar e 0,215 bar, nessa ordem. A densidade do petróleo foi de $0,8675 \mathrm{~g} / \mathrm{cm}^{3}$. A recuperação especial obteve uma recuperação de 4,46 \%, sendo que até o décimo segundo VP foi injetado somente o tensoativo aniônico SB. As pressões inicial e final foram de 0,300bar e 0,330bar, nessa ordem. A partir do décimo terceiro até o décimo sétimo VP, injetou-se novamente a água salina, onde as pressões inicial e final foram de 0,315bar e 0,260bar, respectivamente. O fator de recuperação foi de $0,24 \%$, ou seja, praticamente não houve petróleo deslocado. A recuperação total foi de $12,41 \%$.

A Figura 4 mostra o fator de recuperação com o VP de água salgada e EDTA.

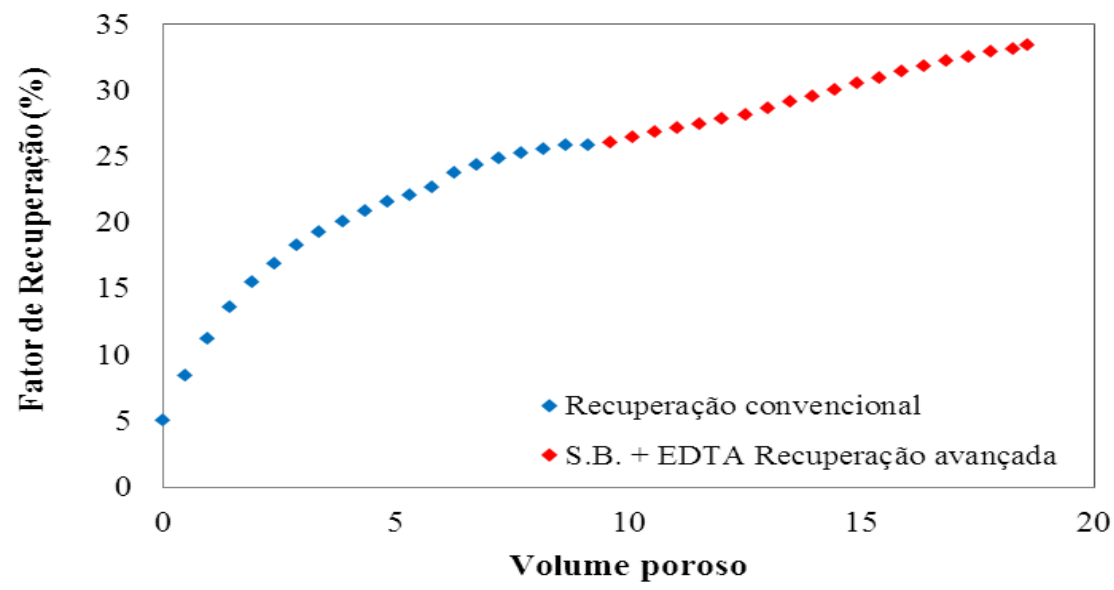

Figura 4 - Relação entre o fator de recuperação e o volume poroso utilizando o EDTA com o tensoativo SB na recuperação avançada. 


\section{9 a 22 de outubro de 2014 \\ Florianópolis/SC}

Analisando a Figura 4, percebe-se que o fator de recuperação na recuperação convencional foi de 26,09\% com a injeção de 10 VP de solução salina. Já a recuperação avançada obteve um fator de recuperação de 7,32\% com a injeção de 8 VP de solução micelar com EDTA a 0,01M. A recuperação total foi de $33,41 \%$.

Os resultados obtidos nesses experimentos foram inferiores ao resultado encontrado por Luo et al. (2013) na recuperação simples com pressão altíssima de aproximadamente 35bar. Eles conseguiram 43,74\% IOIP. Porém, na recuperação avançada todos os experimentos realizados nesse trabalho foram superiores ao resultado encontrado por Luo et al. (2013) utilizando gases $\left(30 \%\right.$ de $\mathrm{CO}_{2}$ com $70 \%$ de $\mathrm{N}_{2}$ ) alternadamente com água obtendo 3,58\% IOIP.

Luo et al. (2013) também realizaram outro experimento no qual a recuperação simples obteve um fator de recuperação de 44,82\% IOIP que foi superior aos encontrados nos experimentos nesse trabalho. Eles usaram um sistema com pressão altíssima de aproximadamente 35bar. Na recuperação avançada, Luo et al. (2013) utilizaram o $\mathrm{CO}_{2}$-WAG (water-alternating-gas) e conseguiram 9,43\% IOIP. Esse resultado foi semelhante aos resultados dos experimentos feitos nesse trabalho mostrados na Figura 2 e na Figura 4 de 7,54\% e 7,32\%, respectivamente. Porém, o fator de recuperação da Figura 3 foi inferior aos dos demais experimentos.

Hezave et al. (2013) utilizaram o líquido iônico cloreto de 1-dodecil-3-metilimidazol $\left(\mathrm{C}_{12} \mathrm{mimCl}\right)$ a uma concentração de $1000 \mathrm{ppm}$ preparado com água destilada e obtiveram 52\% OOIP na recuperação simples. Esse valor foi superior aos encontrados nesse trabalho. Todavia, eles conseguiram 3\% OOIP na recuperação avançada. Esse fator de recuperação foi inferior a todos os encontrados nesse trabalho.

Eles aumentaram a concentração do líquido iônico para 2500ppm e como resultado a recuperação simples foi de $42 \%$ OOIP e a especial foi de $7 \%$ OOIP. Esse resultado da recuperação avançada foi semelhante aos obtidos nas Figuras 2 e 4, porém foi superior ao da Figura 3.

Hezave et al. (2013) continuaram utilizando o líquido iônico cloreto de 1-dodecil-3metilimidazol com concentração de $1000 \mathrm{ppm}$ só que dessa vez preparado a partir da solução salina da formação, composto principalmente por $\mathrm{Na}^{+} / \mathrm{K}^{+}$e $\mathrm{Cl}^{-}$, e chegaram a $52 \%$ OOIP na recuperação convencional. Já na avançada, o fator de recuperação foi de $6 \%$ OOIP; resultado inferior aos encontrados nas Figuras 2 e 4, porém superior ao valor encontrado na Figura 3.

\section{CONCLUSÃO}

De acordo com os fatores de recuperação obtidos nos três experimentos, podem-se obter algumas conclusões importantes. No experimento no qual se utilizou somente o tensoativo aniônico $\mathrm{SB}$, o fator de recuperação foi maior do que quando foi usada a solução salina. A adição de sais ao fluido de injeção na recuperação especial aumenta o fator de recuperação. $O$ experimento realizado com o EDTA mostrou que essa substância, quando adicionada ao tensoativo, não aumenta a porcentagem de óleo recuperado, pois ao usar somente o tensoativo SB obteve-se 7,54\% e ao adicionar o EDTA a 0,01M, o fator de recuperação foi de 7,32\%. Os experimentos foram realizados a 
pressões baixas, e, mesmo assim, os fatores de recuperação na etapa da recuperação avançada foram semelhantes e, algumas vezes, superiores a de outros trabalhos.

\section{AGRADECIMENTOS}

Os autores agradecem a UFERSA e UFRN, pelo suporte técnico na realização do trabalho, e ao PRH(56)ANP pelo suporte financeiro.

\section{REFERÊNCIAS}

AHMADI, M. A.; ARABSAHEBI, Y.; SHADIZADEH, S. R.; BEHBAHANI, S. S. Preliminary evaluation of mulberry leaf-derived surfactant on interfacial tension in an oil-aqueous system: EOR application. Fuel, v. 117, p. 749-755, 2013.

AHMADI, M. A.; SHADIZADEH, S. R. Implementation of a high-performance surfactant for enhanced oil recovery from carbonate reservoirs. J. of Petroleum Science and Eng., v. 110, p. 66-73, 2013.

CHEN, L.; ZHANG, G.; GE, J.; JIANG, P.; TANG, J.; LIU, Y. Research of the heavy oil displacement mechanism by using alkaline/surfactant flooding system. Colloids and Surfaces A: Physicochem. and Eng. Aspects, v. 434, p. 63-71, 2013.

DALTIN, D., Tensoativo: química, propriedades e aplicações. 1. Ed. São Paulo: Blucher, 2011.

HEZAVE, A. Z.; DOROSTKAR, S.; AYATOLLAHI, S.; NABIPOUR, M.; HEMMATEENEJAD, B. Investigating the effect of ionic liquid (1-dodecyl-3-methylimidazolium chloride ([C12mim] [Cl])) on the water/oil interfacial tension as a novel surfactant. Colloids and Surfaces A: Physicochem. and Eng. Aspects, v. 421, p. 63-71, 2013.

KHOSRAVANI S.; ALAEI, M.;RASHIDI, A.M.; RAMAZANI, A.; ERSHADI M. O/W emulsions stabilized with g-alumina nanostructures for chemical enhanced oil recovery. Materials Research Bulletin, v. 48, p. 2186-2190, 2013.

LUO, P.; ZHANG Y.; HUANG, S. A promising chemical-augmented WAG process for enhanced heavy oil recovery. Fuel, v. 104, p. 333-341, 2013.

PEI, H.; ZHANG, G.; GE, J.; ZHANG, L.; WANG, H. Effect of polymer on the interaction of alkali with heavy oil and its use in improving oil recovery. Colloids and Surfaces A: Physicochem. and Eng. Aspects, v. 446, p. 57-64, 2014.

THOMAS, J. E. Fundamentos de Engenharia do Petróleo. Interciência: Petrobras, Rio de Janeiro RJ, 2001.

YIN, D-Y; ZHANG, X-R. Evaluation and research on performance of a blend surfactant system of alkyl polyglycoside in carbonate reservoir. J. of Petroleum Science and Eng., v. 111, p. 153-158, 2013. 